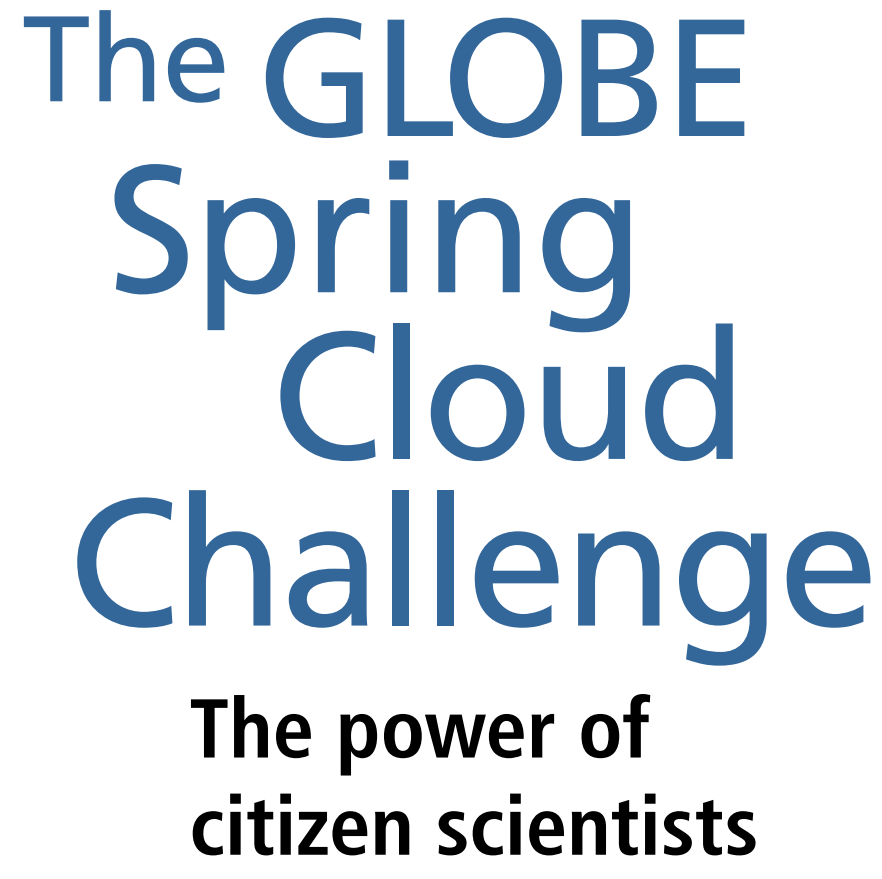

Adapted from "Clouds Around the World: How a Simple Citizen Science Data Challenge Became a Worldwide Success," by Marilé Colón Robles (Science Systems and Applications, Inc., and NASA Langley Research (enter), Helen M. Amos, J. Brant Dodson, Jeffrey Bouwman, Tina Rogerson, Annette Bombosch, Lauren Farmer, Autumn Burdick, Jessica Taylor, and Lin H. Chambers. Published online in BAMS, July 2020. For the full, citable article, see DOI:10.1175/ BAMS-D-19-0295.

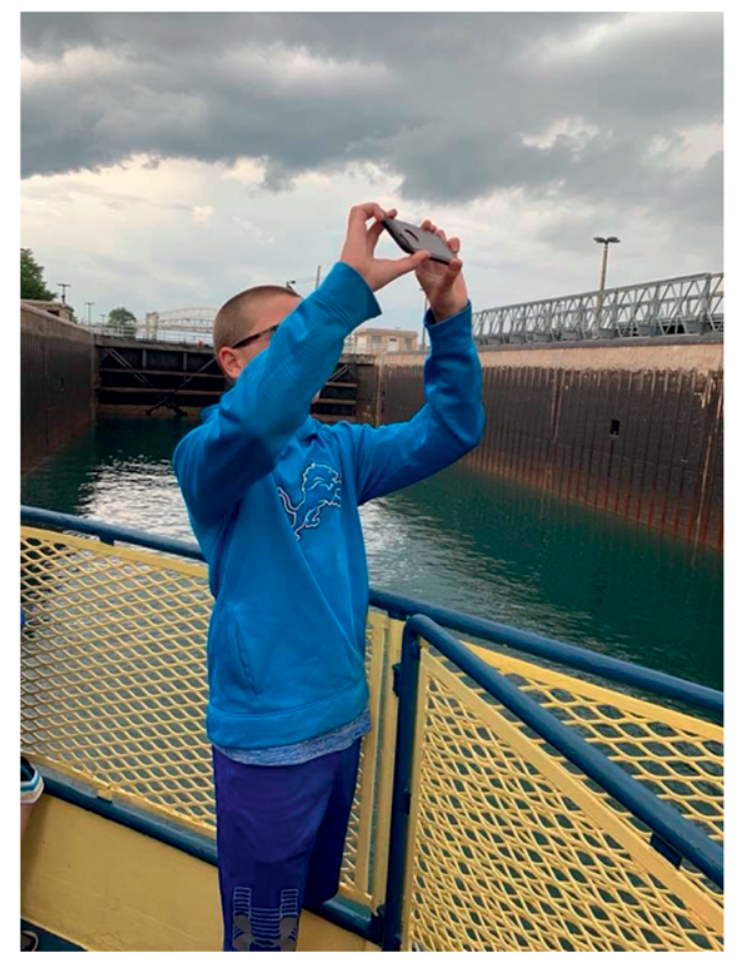

$\mathrm{T}$ he 2018 Global Learning and Observations to Benefit the Environment (GLOBE) Clouds Spring Data Challenge was a citizen science event that collected a total of 55,639 ground-based visual cloud observations by engaging participants from more than 15,000 locations in 99 countries, on every continent. Participants of this Spring Cloud Challenge were asked to take up to 10 cloud observations per day from 15 March through 15 April 2018.

Since its inception in 1995, the GLOBE Program and its 120 formally participating nations have leveraged the expertise of scientists and educators, developing a worldwide community to better understand, sustain, and improve Earth's environment. GLOBE is sponsored by NASA and is supported by the U.S. Department of State, the National Science Foundation, and NOAA. The University Corporation for Atmospheric Research (UCAR) manages the GLOBE Program's Implementation Office.

Everyone collecting data through GLOBE follows the same scientific procedure and instrument specifications, as necessary. GLOBE allows for a continuous flow of citizen science data collected year round. Teachers use events like the Spring Cloud Challenge 
to encourage students and, in turn, to create schoolwide events that go beyond the school year.

If a citizen scientist makes an observation at the same time and location as the satellite's measurements, NASA sends a personalized email to the participant. The email contains

Ground-based visual cloud observations collected during the Spring Cloud Challenge (15 Mar-15 Apr 2018) color coded based on the world region as defined by the GLOBE program: North America (NA) in yellow, Latin America (including South America) and the Caribbean (LAC) in orange, Europe and Eurasia (EEA) in teal, Near East and North Africa (NENA) in red, Africa (AFR) in purple, Asia and the Pacific (ASP) in green, Drake Passage (DRK) in blue, and all other areas in gray. (a) Total observations received through GLOBE's pen-and-paper mode of data collection. (b) Observations received through the GLOBE Observer mobile app.

Pen-and-paper mode of data collection $=15,431$
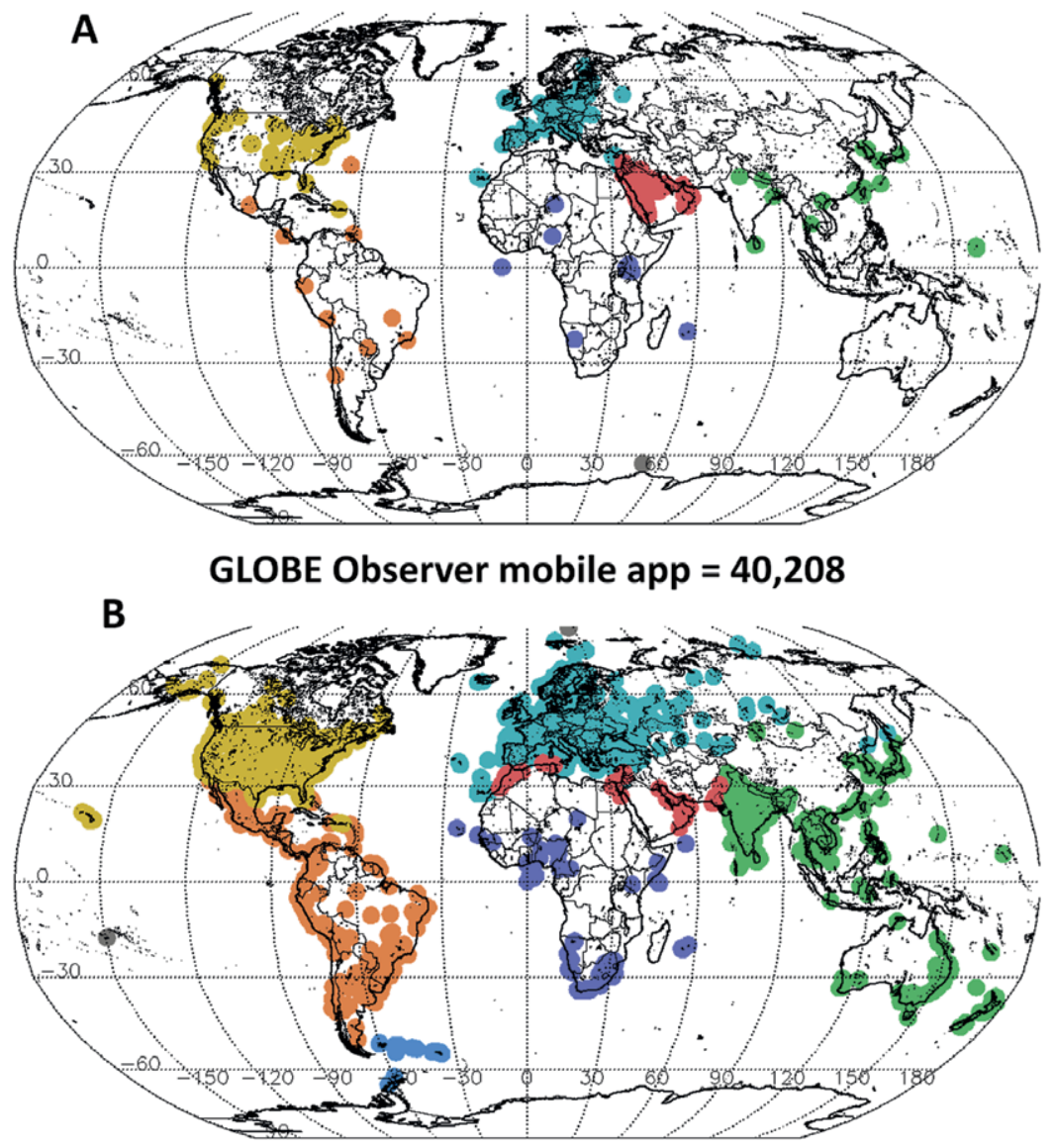

a link to a satellite match table displaying the person's observations alongside the satellite collocation results, which include the actual satellite images of the collocation results, enabling participants to learn how satellites study Earth's atmosphere. These collocations of ground-based visual cloud observations and satellite-derived observations, like all data collected through GLOBE, are freely available for each latitude and longitude on the GLOBE Observer website. ${ }^{1}$ The GLOBE Program has received more than 550,000 citizen science ground-based visual cloud observations since 2017 from 34,000 schools and more than 140,000 citizen scientists.

Each cloud observation includes information on cloud fraction, obscuration (e.g., sand, fire, heavy rain or snow), and surface conditions (e.g., snow or ice on the ground), plus additional optional observations. In addition to building community awareness and public engagement, GLOBE Clouds data are being used in satellite validation, contrail investigations, comparisons of cloud types in different parts of the world, and to evaluate the NASA Goddard Earth Observing System (GEOS) model, investigate marine haze over the Southern Ocean, and augment sparse ground-based monitoring data on dust events.

The robustness of these groundbased visual cloud observations was borne out during the total solar eclipse over North America in 2017. Through the use of a Monte Carlo-style sampling test, the utility of an analysis methodology that takes advantage of the large volume of observations to compensate for variability in reporting among volunteer citizen scientists was demonstrated. The Spring Cloud Challenge generated roughly three times as many observations as that solar eclipse.

\section{The mobile app}

In 2016, the program debuted the GLOBE Observer mobile app, created to engage citizen scientists, including adults who had participated as students. To ensure accessibility, only data collection that requires no- or low-cost instrumentation is part of the app. Ground-based visual cloud observations, when possible, are collocated 


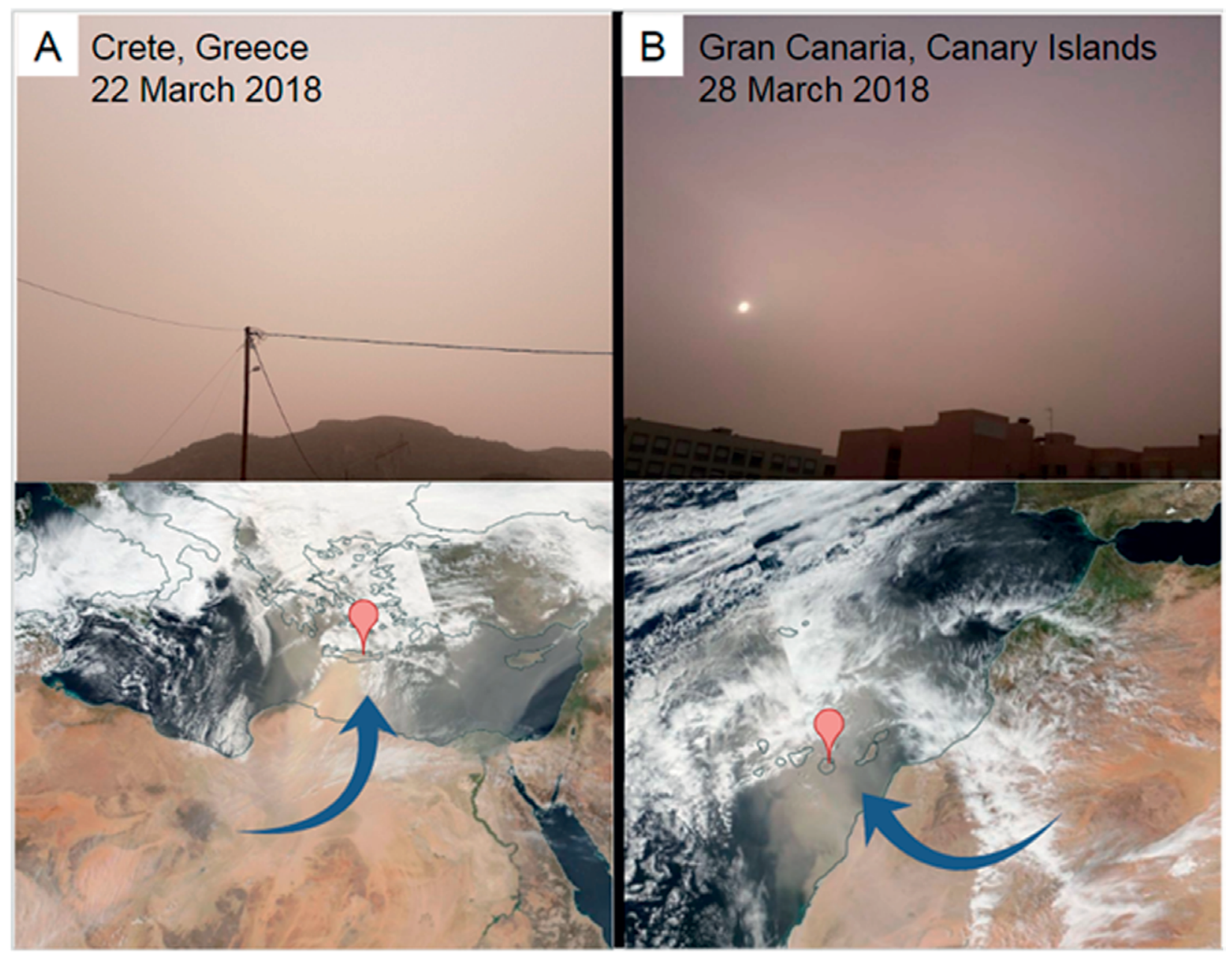

to the nearest overpass times of geostationary satellites (GOES-15, GOES-16, Meteosat-8, Meteosat-11, or Himawari-8), or to Clouds and the Earth's Radiant Energy System (CERES) instruments onboard Aqua and Terra, or the Cloud-Aerosol Lidar and Infrared Pathfinder Satellite Observations (CALIPSO) satellite.

The mobile app contains tutorials explaining data collection and submission. Though the GLOBE app is used by formally GLOBEtrained classroom teachers and students, the bulk of the app users are anonymous volunteer citizen scientists. Globe data collection can also be done using pen and paper by schools around the world.

The GLOBE Observer mobile app guides users to orient their smartphone cameras horizontally, aligned in the cardinal directions, and tilted to a $14^{\circ}$ angle to mimic a conventional sky camera; the app is then programmed to automatically take photographs. This produces a consistent image set across users. At
$\Delta$ * Intense, multiday Saharan dust event in spring 2018 observed (bottom) from space by NASA satellites and (top) from the ground by citizen scientists with the NASA GLOBE Observer mobile app. Image credits: NASA Worldview MODISIAqua and Terra, Suomi NPP, and NASA GLOBE Observer. about 5 min per observation, the observations in the Spring Cloud Challenge alone represent roughly 4,500 h of volunteer time.

Matches to geostationary satellites occur if observations are within $15 \mathrm{~min}$ from half-hourly to hourly data within the GOES field, and 15 min within every hour in locations within the Meteosat and Himawari fields of view. Spatially, the match includes all geostationary data within a $40-\mathrm{km}$ radius circle around the ground observation and includes both an infrared and visible image. During the Spring Cloud Challenge, 33,974 observations (61\%) were collocated to satellite overpasses.

\section{Extending GLOBE's reach}

An initial news release advertising the Spring Cloud Challenge in March 2018 was followed within 5 days by more than 120 news articles published in multiple countries and languages. That led to a surge of observations and broadcast news stories. Further publicity was given 
Total Cloud Observations Submitted to GLOBE (January 2017 - December 2019)

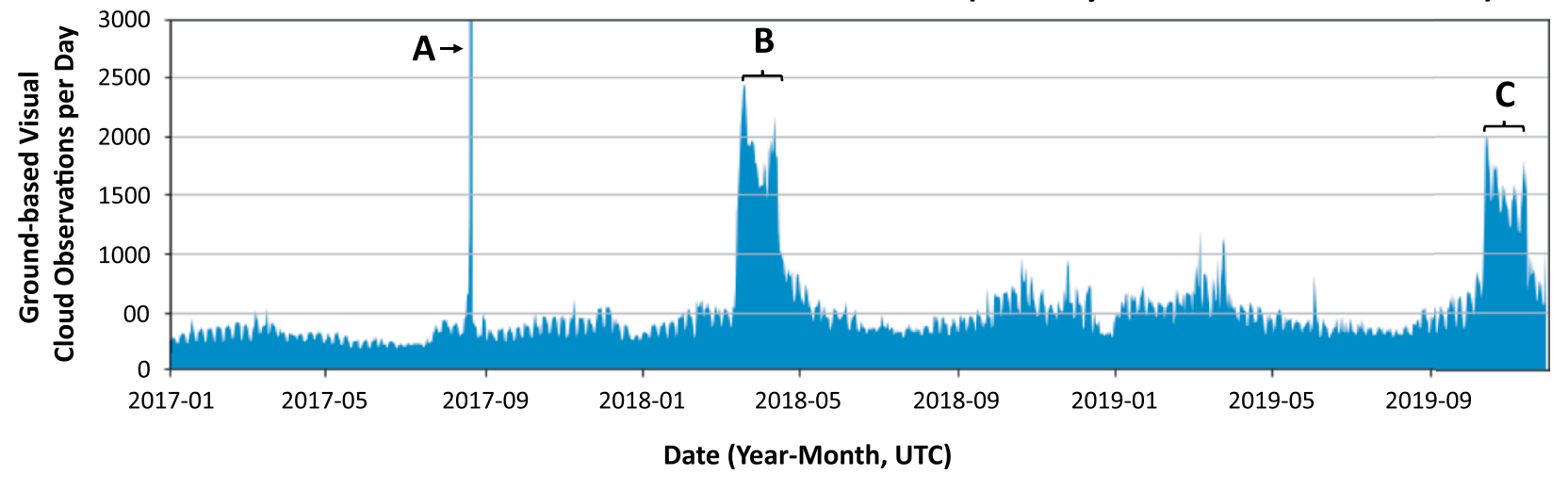

on a French network television station during a popular news program. The Challenge was also highlighted on the WMO's World Met Day and by NASA with live Facebook events. Collectively, these efforts reached more than 3.6 million people, and more than 27,000 new users registered for the mobile app during the challenge period-a 15-fold increase in app users, and a concomitant spike in the amount of data collected.

Even after the Challenge began, frequent communication and outreach motivated participants to submit multiple observations and maintain interest. Later, we thanked the worldwide community in a video featuring multiple NASA scientists and educators congratulating all the observers in multiple languages. Top observers in each region received formal recognition from a NASA scientist.

A special region where data were reported during the data challenge was over the Drake Passage, between the tip of South America and the Antarctic Peninsula. No collocations with geostationary satellites were obtained in the region. Several cruise operators involve their passengers in observing cloud cover using the GLOBE mobile app. The Polar Citizen Science Collective (PCSC; www.polarcollective.org) helps drive this collaboration between the polar tourism industry and the scientific community. Ship operators train interested passengers. This unique, over-the-ocean perspective of cloud and visibility observations can supply unique information about clouds and haze layers near the ocean surface.

\section{Summary}

The data challenge increased the number of observations and extended GLOBE's reach to
$\Delta$ * Total number of ground-based visual cloud observations received through the GLOBE program per day from January 2017 to December 2019. Three big intensive observation periods are evident within the number of observations received per day: the North American total solar eclipse on 21 Aug 2017 (labeled A), the Spring Cloud Challenge (15 Mar-15 Apr 2018; labeled B), and the Fall Cloud Challenge (15 Oct-15 Nov 2019; labeled C).

\section{GLOBE Clouds observation categories}

\section{Observation}

\section{Total cloud cover}

Obscuration (if $>.25 \%$ sky covered)

Sky color (if $<50 \%$ cloud cover)

Sky visibility (if $<50 \%$ cloud cover)

Cloud types by height

Opacity by height

Cloud cover by height

Surface condition

Photographs 
areas that are difficult or too costly to study. The GLOBE Spring Cloud Challenge was also a resounding success in demonstrating the effectiveness of engaging and training citizen scientists from around the world. Recruitment was facilitated by the simplicity of the task.

In late 2019, a second citizen science challenge, the Fall Cloud Challenge: What's Up in

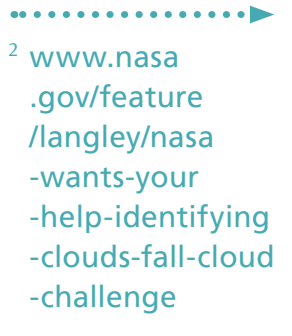

Your Sky?², was also successful. The GLOBE Clouds team will next launch regional calls for photographs of dust events during peak seasons, with emphasis on observations from the southwestern United States and from high-latitude locations. The Spring and Fall Cloud Challenges augur well for similar approaches to future challenges in science. $\bullet \bullet$

\section{METADATA}

BAMS: What would you like readers to learn from this article?

\section{Marilé Colón Robles (Science Systems and Applications, Inc., and NASA Langley Research Center):}

The general public, from students to teachers to families, are very eager to be part of finding ways to learn more about our planet. Simple steps, focusing on what they already have, and constant communication are key to keep them engaged. Highlighting their role in the projects and how important their participation is in the project is key to being successful. The strength of citizen science data is the volume and location of the observations. Researchers need to consider new techniques for analyzing the data that take advantage of those strengths.

BAMS: How did you become interested in the topic of this article?

MCR: My passion as a Latina woman in science is to show students and underrepresented groups how they have what it takes to be successful in STEM (Science, Technology, Engineering, Mathematics). Being part of NASA's largest and longest-lasting citizen science project about the environment gives me a unique opportunity to interact with over 200,000 students, teachers, and members of the general public from more than 120 countries, and the chance to show them their strengths, their capabilities, and their impact in research.

BAMS: What surprised you the most about the work you document in this article?

MCR: We had set out to do a simple engagement event that asked people to collect as many cloud observations as they could through The GLOBE Program with the promise of a NASA scientist reading the names of the top observers in a congratulatory video. When the announcement went out, it was like wildfire and we had to quickly adjust and make shareables, constantly message the public, and increase the size of the servers because so much data were coming in during the period. It was astonishing and amazing to see the willingness people all around the world had to be part of the event. The most special part was to receive comments back from the top observers and how amazed they were for the recognition and that they were part of the solution. One of the most touching emails was from one of the winners of the 2019 Fall Cloud Challenge saying that she was a mom who didn't know a lot about science, but her daughter would remind her to go out and collect her cloud observations and, thanks to that, she was one of the winners!

BAMS: What was the biggest challenge you encountered while doing this work?
MCR: You have to be quick on your feet, brainstorm multiple solutions, and with a trusted team, come up with the best idea possible in a very short amount of time. It creates a sense of teamwork and a constant reminder that you can learn from anyone, no matter their background or specialty.

BAMS: How did you follow up your research?

MCR: We put together the 2020 Community Cloud Challenge (15 July-15 August 2020) in a very challenging time due to the global pandemic. We wanted people to really know how they are the most important component of any project. Their safety and well-being is number one, and we adapted this third challenge by offering ways for families to engage. We created the Cloud Activity Tracker for people to keep track of their participation through engaging, learning, creating, or observing. We also had weekly videos featuring NASA subject matter experts and GLOBE educators to showcase different aspects of clouds, their importance in research, and an at-home activity anyone could do related to the science topic (https://observer.globe.gov/es ldo-globe-observer/challenges /community-cloud-challenge-2020). We have received over 22,000 observations from more than 79 countries, resulting in more than 23,000 satellite matches. Given that we are in a global pandemic, this has been a success. 


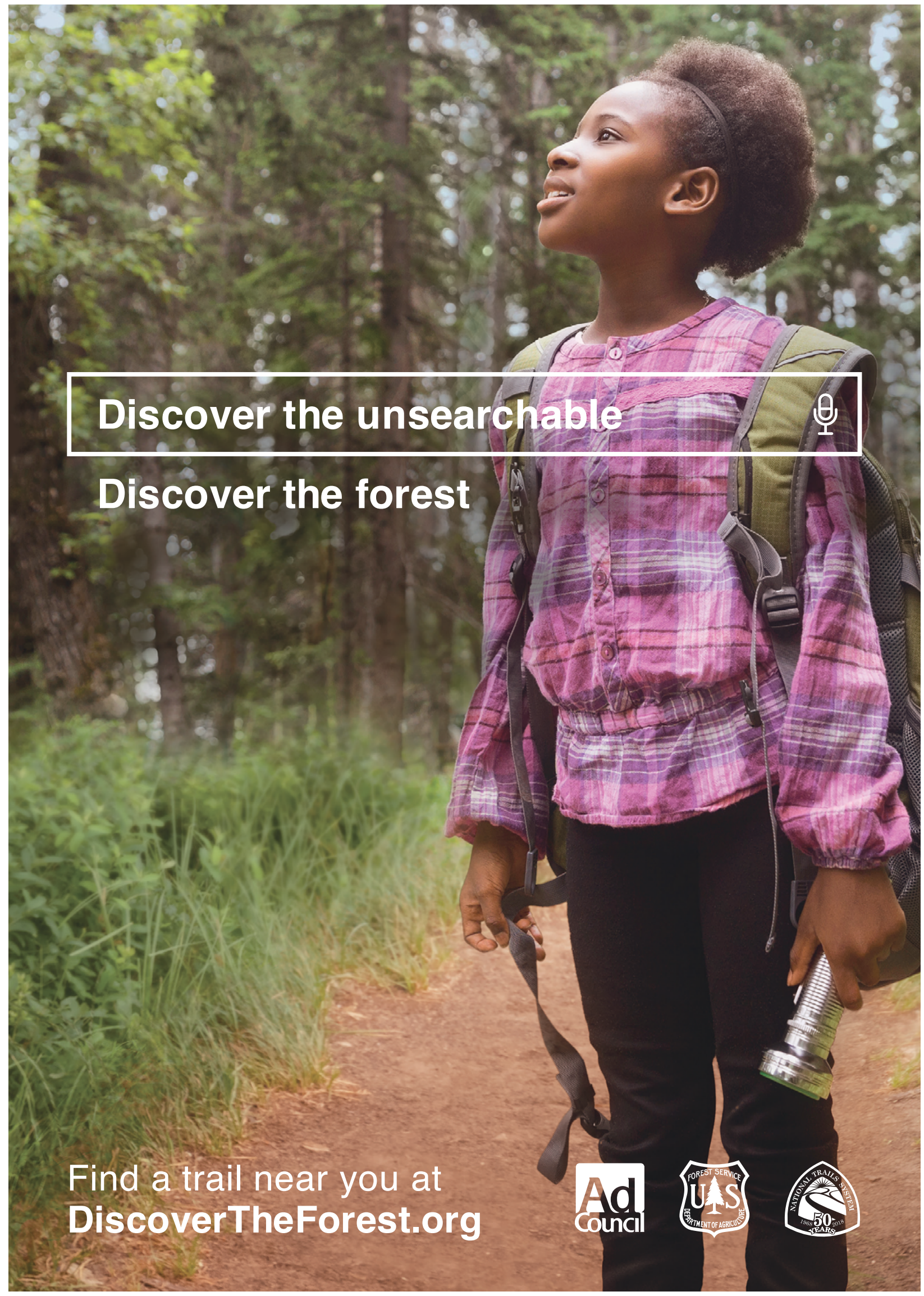

